\title{
Accurate Hysteresis Loops Calculation Under the Frequency Effect Using the Inverse Jiles- Atherton Model
}

\author{
Ibtissem Belgasmi1 ${ }^{1}$ Mourad Hamimid² \\ ${ }^{1}$ Laboratoire de Génie Electrique ‘Université de Med BOUDIAF-BP 166 M'sila 28000, Algérie, \\ ${ }^{2}$ Laboratoire de Physique Des Matériaux, Rayonnements et Nanostructures, Université de Bordj Bou Arreridj, 34265, Algérie \\ Corresponding author: M. HAMIMID (e-mail: mourad.hamimid@univ-bba.dz)
}

\begin{abstract}
In this present work, an attempt has been made to improve the shape of hysteresis loops as well as an accurate calculation of the iron losses under the frequency effect. The inverse Jiles-Atherton model is extended to describe the magnetization of ferromagnetic material behavior in dynamic regime. A new formulation of the magnetic effective field is used which consists of modifying the expression of the excess field to take correctly into account the moving domain walls effect. The new proposed expression of the effective field allows a good representation of the magnetic hysteresis behavior regarding the frequency increase. To validate this proposal approaches a measured and modeled hysteresis loops for different frequencies are compared.
\end{abstract}

INDEX TERMS Effective field, Excess losses, Frequency effect, Jiles-Atherton Model, Hysteresis, Iron losses.

\section{INTRODUCTION}

Tor an optimal design of the magnetic cores
constituting the essential element in the electromagnetic conversion of electrical energy devices, the correct determination of iron losses is indispensable. These losses are normally estimated using several approaches such as Steinmetz's formula [1], Bertotti's loss separation method [2]-[3], or mathematical hysteresis models [4]. The diversity of these methods shows the difficulty encountered in the accurate determination of these losses. In ferromagnetic materials these losses are represented by the area of the hysteresis loops. Several approaches are available for the modelling of ferromagnetic hysteresis. The Jiles-Atherton model [5], is one of the most popular models, due to its relative simplicity and physical backgrounds. The internal magnetic field, i.e. the effective field [6], plays an important role in the description of hysteretic behavior in these materials.

Increasing the frequency of the supply voltages causes the flow of eddy currents induced in ferromagnetic core material. These induced currents produce an additional magnetic field, in opposition to the effective field [7]. Moreover; the source of excess losses can be interpreted as the result of another field also in opposition to the internal field.

In this work, an attempt has been made to improve the shape of hysteresis loops and consequently, iron losses determination under the frequency effect. The inverse Jiles-
Atherton model is extended to describe the hysteresis loops of the soft magnetic material in dynamic regime, including a modification in the effective field. In which the counterfields produced by the eddy currents and excess losses, are given by new expressions. By varying the excess field expression, the shape and the total losses are immediately ameliorated. The obtained results are verified on $3 \% \mathrm{Fe}-\mathrm{Si}$ non-oriented magnetic sheets.

\section{QUASI-STATIC INVERSE JILES -ATHERTON MODEL}

The Modified Inverse Jiles-Atherton model (MIJA) considers the magnetic flux density $B$ as an independent variable [8]-[9], is given below by equation (1) with

$H_{e}$ and $M_{a n}$ are, respectively, the effective field and the anhysteretic magnetization, the directional parameter $\delta$ is taking the value +1 if $d B / d t>0$ and -1 if $d B / d t<0$.

$a, \alpha, c, k$ and the saturation magnetization $M_{s}$ are the five model parameters which have to be determined from measured major hysteresis loops.

$$
\frac{d M}{d B}=\frac{\xi}{\mu_{0}(k \delta+(1-\alpha) \xi)}
$$

with the following complementary relationships :

$$
\xi=M_{a n}-M+k c \delta \frac{d M_{a n}}{d H_{e}}
$$




$$
\begin{gathered}
M_{a n}=M_{s}\left(\operatorname{coth} \frac{H_{e}}{a}-\frac{a}{H_{e}}\right) \\
H_{e}=H+\alpha M \\
\frac{d M_{a n}}{d H_{e}}=\frac{M_{s}}{a}\left(1-\operatorname{coth}^{2} \frac{H_{e}}{a}+\left(\frac{a}{H_{e}}\right)^{2}\right)
\end{gathered}
$$

The magnetic field in the quasi static regime is obtained by

$$
H_{\text {stat }}=\frac{B}{\mu_{0}}-M
$$

In this work 3\% Fe-Si non-oriented magnetic sheets are used These sheets are characterized by $0.35 \mathrm{~mm}$ thick laminations, $15 \mathrm{~mm}$ width and $7650 \mathrm{~kg} / \mathrm{m} 3$ mass density. The measurement of the hysteresis loops is performed by using Epstein frame, this device plays the role of transformer with primary coils of 640 turns and secondary coils of 320 turns. The mean length of the magnetic core is $147 \mathrm{~mm}$.

Table I presents these parameters identified in quasi-static regime $(10 \mathrm{~Hz}$, this is the stable lower limit of our generator), by using the same procedure cited as in [8]. Figure 1 shows the measured and the modelled hysteresis loops.

TABLE I. Major Hysteresis Loop Parameters

\begin{tabular}{ll}
\hline \hline J-A model parameters & Quasi-Static values \\
\hline$M_{s}(\mathrm{~A} / \mathrm{m})$ & $1.19 \cdot 10^{6}$ \\
$a(\mathrm{~A} / \mathrm{m})$ & 56.14 \\
$k(\mathrm{~A} / \mathrm{m})$ & 60.10 \\
$\alpha$ & $1.95 \cdot 10^{-4}$ \\
$c$ & $1.33 \cdot 10^{-2}$ \\
\hline \hline
\end{tabular}

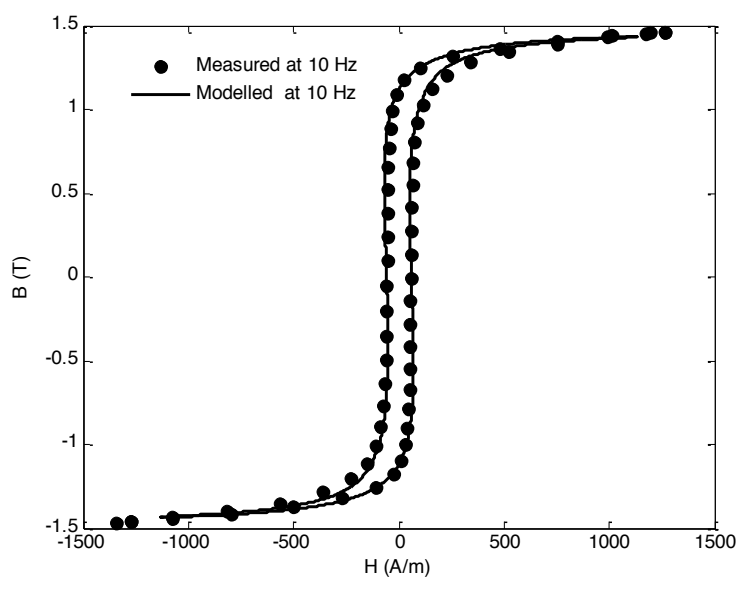

FIGURE 1. Modelled and measured hysteresis loops

\section{DYNAMIC EXTENSION OF THE MIJA MODEL}

The MIJA hysteresis model presented above describes accurately the quasi-static magnetization process of the domain structure. In the previous research [9], an attempt was made to include the effect of dynamic regime in MIJA by adding two reaction fields resulting from eddy currents and excess losses in the expression of the effective field. This dynamic model gives acceptable results but the shape of the hysteresis loop is not well represented. In this work, we introduce a new expression of the excess losses based on the viscous-type equation describing the time lag between the flux density $B(t)$ and the applied field $H(t)$ [10-12] as given below in equation (4).

$$
\frac{d B(t)}{d t}=\frac{\delta}{\mathrm{g}(B)}\left|H(t)-H_{\text {stat }}(B)\right|^{v}
$$

The $H_{\text {stat }}$ represents the static hysteresis field calculated using the quasi-static inverse Jiles-Atherton model given in section 2. $v$ is a parameter for a given material, by varying this parameter along some parts of the loop we can control the shape and total loss [12]. $1 / g(B)$ is called dynamic magnetic resistivity and $g(B)$ in general is a function of $B[10]$. The most prevalent expression of function $g(B)$ is given by [13]

$$
g(B)=\frac{C_{2}}{\left(1-\left[B(t) / B_{\max }\right]^{2}\right)}
$$

$C_{2}$ is constant for given material and $B_{\max }$ is the maximum induction reachable in calculations and experiment. Using (4) and (5) the total field is given as

$$
H(t)=H_{\text {stat }}(t)+\delta\left|\operatorname{g}(B) \frac{d B(t)}{d t}\right|^{1 / v}
$$

The second term in (6) represents the excess field $H_{\text {exc }}$ expression whose value is proportional to $|d B(t) / d t|^{1 / v}[11]$. By using this expression of excess field $H_{e x c}$, the volumetric energy density dissipated per cycle and per unit volume in the interval of time $\Delta t$ due to excess losses is given as

$$
W_{e x c}=\int_{\Delta t}\left|\operatorname{g}(B) \frac{d B(t)}{\Delta t}\right|^{1 / v} \frac{d B}{\Delta t} \Delta t
$$

In other hand, this volumetric energy density is given in [9, 14] by the following expression

$$
W_{e x c}=C_{e x c} \int_{\Delta t}\left|\frac{d B(t)}{\Delta t}\right|^{1 / 2} \frac{d B}{\Delta t} \Delta t
$$

The comparison between (7) and (8) gives an overview of the value of $v$. It is clearly visible when $g(B)$ is constant and the 
exponent $v=2$, the two expressions which give the excess losses coincide perfectly.

The magnetic effective field given by (2.c) is modified by introducing the effect of the eddy currents and the excess losses and is given by

$$
H_{e}=\underbrace{H+\alpha M}_{1}-\underbrace{\left(C_{1} \frac{d B(t)}{d t}+\delta\left|\mathrm{g}(B) \frac{d B(t)}{d t}\right|^{1 / v}\right)}_{2}
$$

In this expression the first term is the same as given by (2.c) in the quasi-static model, the second term presents the contribution of the induced currents and excess losses. In the second term $C_{1} d B(t) / d t$ represents the field caused by eddy currents reaction.

Where $C_{I}$ is a coefficient related to the physical and geometrical parameters of material.

The coefficients $C_{1}$ and $C_{2}$ in most cases are not known for all materials, in such cases, this difficulty is overcome by identification of these coefficients in dynamic regime.

It has to be noted that the statistical loss-separation theory proposed by G.Bertotti, [2] does not hold if the skin effect not negligible.

\section{RESULTS AND DISCUSSION}

In the first step the five parameters of Jiles-Atherton model in quasi-static regime $(10 \mathrm{~Hz})$ are identified and they are presented in Table I. To obtain the coefficients $C_{l}$ and $C_{2}$ in dynamic regime we use the experimental results of $100 \mathrm{~Hz}$ by minimizing the error between the measured magnetic field and the modelled one. The stochastic method based on the genetic algorithm is used. This technical is implemented in MATLAB software using the GA Toolbox. The objective function to be minimized is given by

$$
e r r=\frac{\sqrt{\sum_{i=1}^{N}\left(H_{\text {meas }}^{i}-H_{\text {mod }}^{i}\right)^{2}}}{N}
$$

Where:

$H_{\text {meas }}$ and $H_{\text {mod }}$ are respectively the measured and the modelled magnetic field, $N$ is the number of measured points. Table II, shows the values of the new parameters $C_{1}$ and $C_{2}$.

TABLE II. New Dynamic Parameters

\begin{tabular}{ll}
\hline \hline Quasi-Static values & New parameters \\
\hline $29 \cdot 10^{-3}$ & $C_{l}(\mathrm{~m} / \Omega)$ \\
$66 \cdot 10^{-3}$ & $C_{2}(\mathrm{~A} / \Omega)^{1 / 2}$ \\
\hline \hline
\end{tabular}

These new dynamic parameters are obtained by using the modified effective field expression given by (9) and by choosing the exponent $v=2$. The dynamic loops are calculated for the frequencies $50 \mathrm{~Hz}$ and $100 \mathrm{~Hz}$ are presented in Figs. 2 and 3. Good agreement is obtained compared with measurement.

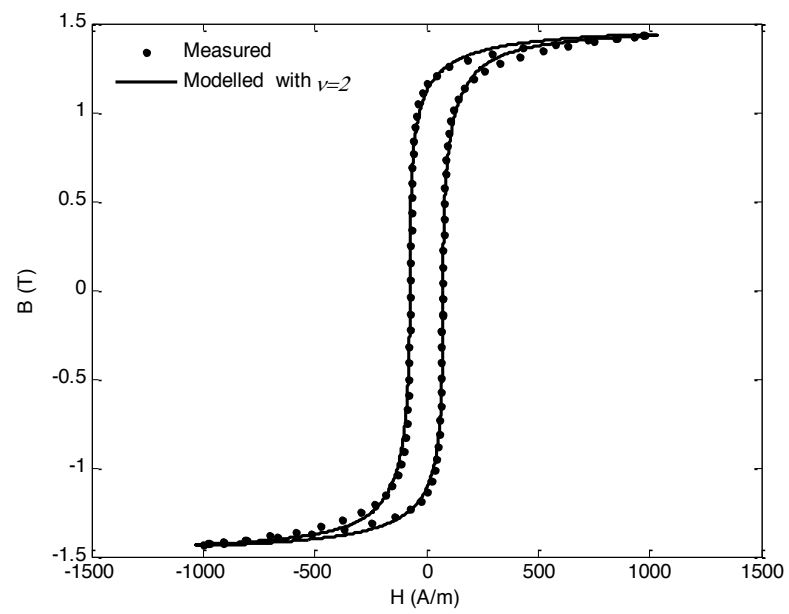

FIGURE 2. Modelled and measured hysteresis loops at $50 \mathrm{~Hz}$

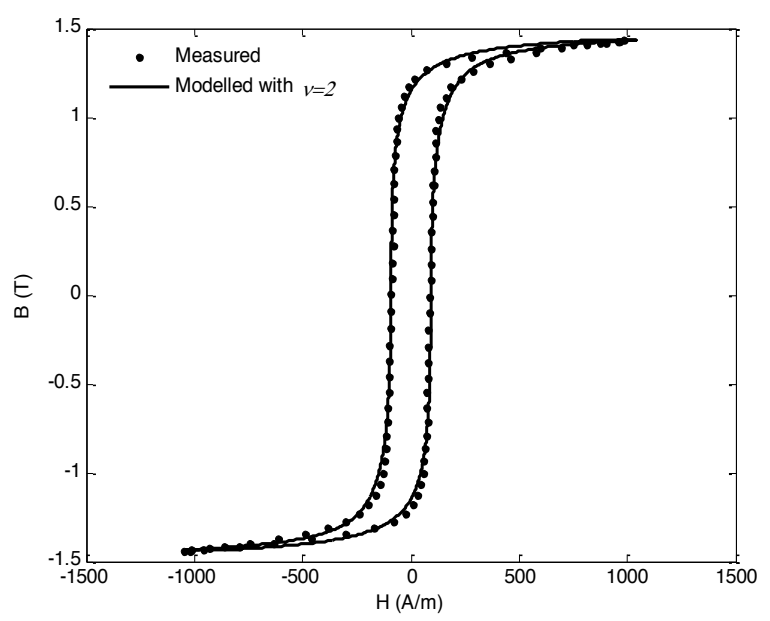

FIGURE 3. Modelled and measured hysteresis loops at $100 \mathrm{~Hz}$

When the frequency is increased more than $100 \mathrm{~Hz}$ a swelling appears on the shape of the measured hysteresis loops. This swelling becomes more and more visible for higher frequencies as shown in Figs 4-6. Figs. 4-6, show that when we use $v=2$, dynamic hysteresis loops modelled for increased frequency do not fit measured ones. 


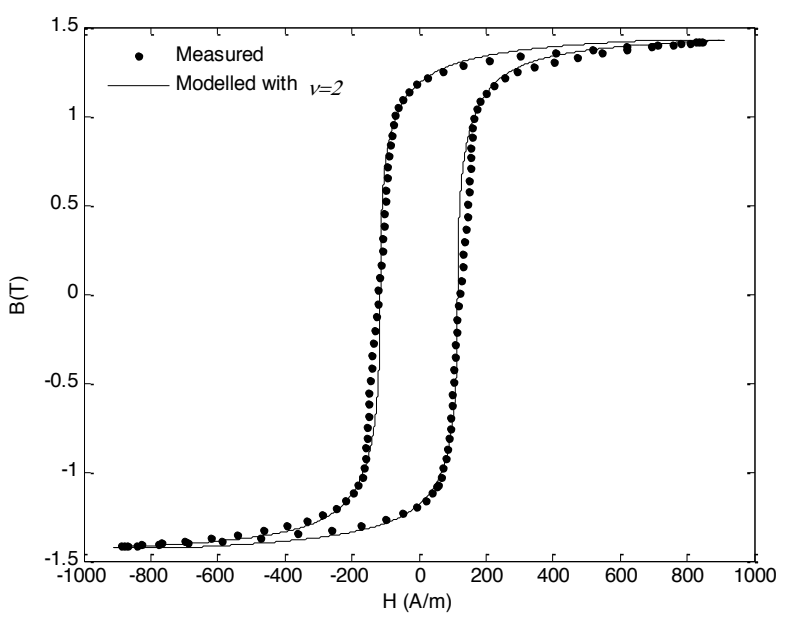

FIGURE 4. Modelled and measured hysteresis loops at $200 \mathrm{~Hz}$

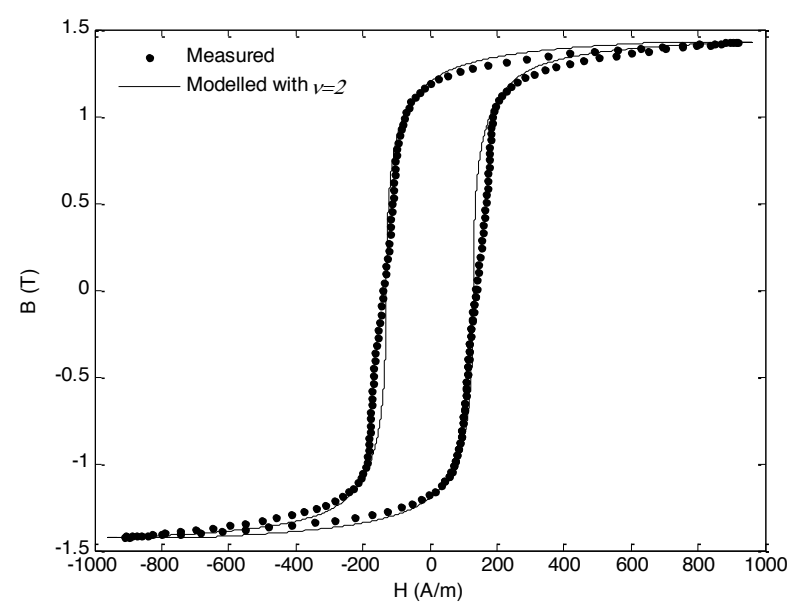

FIGURE 5. Modelled and measured hysteresis loops at $250 \mathrm{~Hz}$

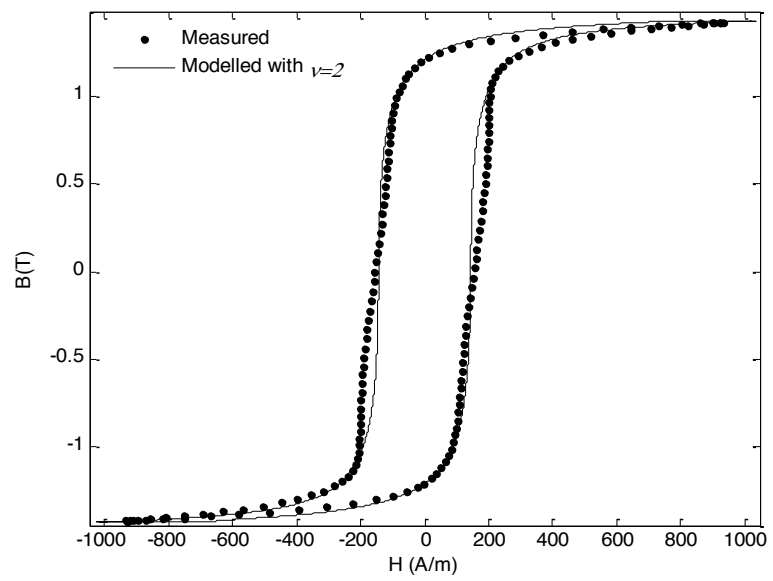

FIGURE 6. Modelled and measured hysteresis loops at $300 \mathrm{~Hz}$
To improve the shape of the modelled hysteresis loops we need to modify the value of $v$ along the parts where swelling appears. The optimal value of the exponent $v$ is given according to the flowing condition

$$
\left\{\begin{array}{c}
\text { if }[\delta(B(t))>0 \text { and } \delta(B(t))<0.8] \\
\text { then } v=1.3 \\
\text { else } \quad v=2
\end{array}\right.
$$

The optimal value when swelling appears is obtained by using the Pattern Search (PS) in MATLAB Optimization toolbox.

By using this condition for $v$, the modelled and measured dynamic hysteresis loops are quite similar as shown in Figs.7-9.

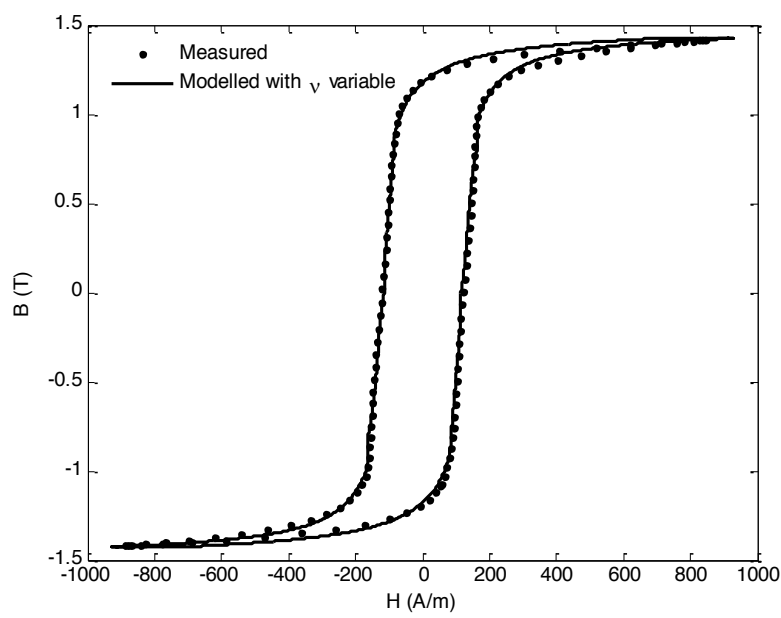

FIGURE 7. Modelled and measured hysteresis loops at $200 \mathrm{~Hz}$ with $v$ variable

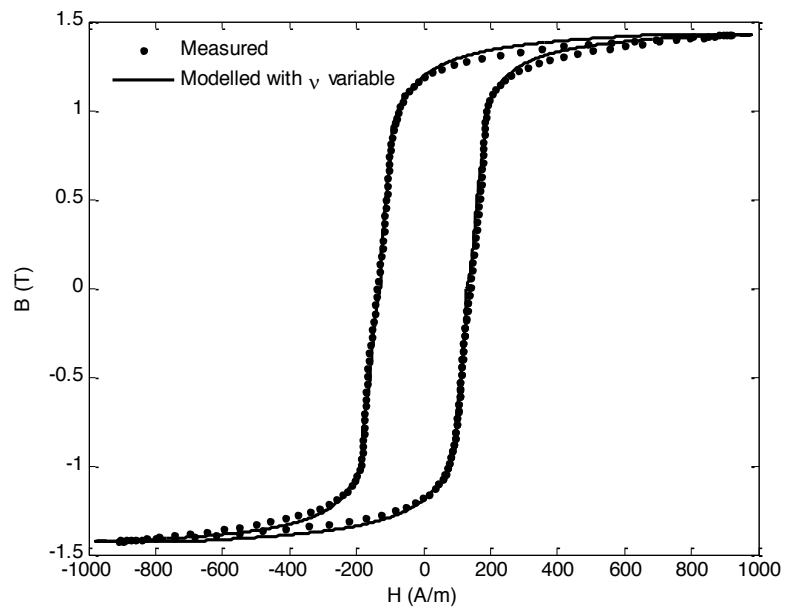


FIGURE 8. Modelled and measured hysteresis loops at $250 \mathrm{~Hz}$ with $v$ variable

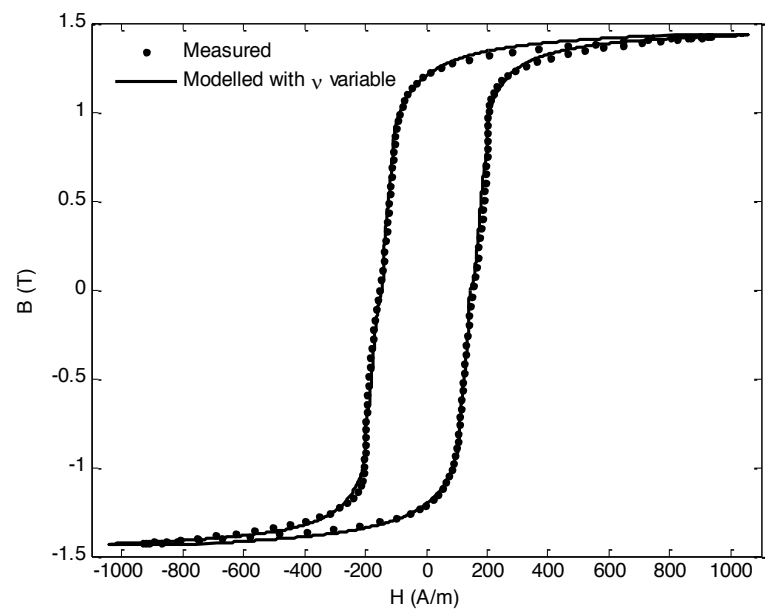

FIGURE 9. Modelled and measured hysteresis loops at $300 \mathrm{~Hz}$ with $v$ variable

Figure 10, shows the power losses modelled and measured for various frequencies, and figure 11 shows the difference between the modelled and measured values. It is clear that the computed power losses with $v$ variable compared to experiment give accurate results than those evaluated by using $v=2$.

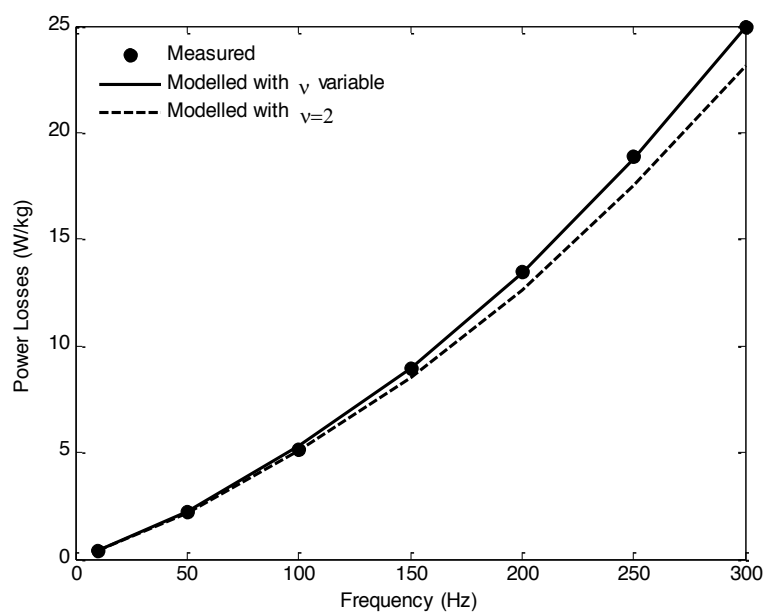

FIGURE 10. Evolution of power losses modelled and measured at different frequencies

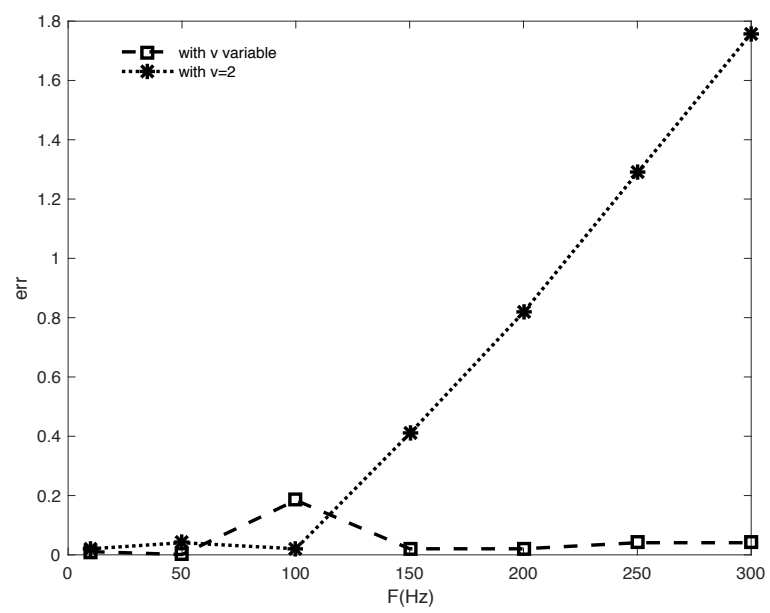

FIGURE 11. Evolution of the errors between modelled and measured power losses at different frequencies

\section{CONCLUSION}

The proposed modification of effective field in dynamic regime, using modified inverse Jiles-Atherton model, is applied for the description of either quasi-static or dynamic magnetization. This modification is based on the introduction of two additional counter fields caused by eddy currents and excess losses in the effective field expression. The validity of the proposed model depends on the exponent $v$. By using appropriate value of $v$ in different regions, the shape of the hysteresis loops is improved. The validity of the model is applied for 3\% Fe-Si non-oriented magnetic sheets and accurate results are obtained.

\section{REFERENCES}

[1] Chas. P. Steinmetz, "On the law of hysteresis," American Institute of Electrical Engineers Transactions., vol. IX, no. 1, pp. 1-64, Jan. 1892.

[2] G.Bertotti, "General Properties of Power Losses in Soft Ferromagnetic materials," IEEE Trans. on Magn., vol.24, no.1, pp. 621-630, 1988.

[3] G. Bertotti, "Hysteresis in Magnetism," Academic Press, San Diego, 1998.

[4] I. D. Mayergoyz, "Mathematical models of hysteresis and their applications," Academic Press, 2003.

[5] D. C. Jiles and D. L. Atherton, "Theory of ferromagnetic hysteresis," J. Magnetism and Magnetic Materials. vol.61, pp. 48-60, 1986.

[6] H.W.L. Naus, "Ferromagnetic hysteresis and the effective field," IEEE Trans. Magn. vol. 38, no.5, pp. 3417-3419, 2002.

[7] J. Szczygłowski, "Influence of eddy currents on magnetic hysteresis loops in soft magnetic materials", J. Magn. Magn. Mater. 223 (2001), pp. $97-102$.

[8] M. Hamimid, M. Feliachi and S. M. Mimoune, "Modified JilesAtherton model and parameters identification using false position method," Physica B : Condensed Matter, Vol. 405, (8), pp. 19471950, 15 April 2010.

[9] M. Hamimid, S. M. Mimoune and M. Feliachi, "Hybrid magnetic field formulation based on the losses separation method for modified dynamic inverse Jiles-Atherton model," Physica B : Condensed Matter, Vol. 406, (14), pp. 2755-2757, 15 July 2011.

[10] Sergey E. Zirka, Yury I. Moroz, Philip Marketos and Anthony J. Moses, "Viscosity-Based Magnetodynamic Model of Soft Magnetic Materials," IEEE Trans. Magn. vol. 42, no.9, pp. 2121-2132, 2006. 
[11] Sergey E. Zirka, Yury I. Moroz, Philip Marketos, and Anthony J. Moses, "A Viscous-Type Dynamic Hysteresis Model as a Tool for Loss Separation in Conducting Ferromagnetic Laminations," IEEE Trans. Magn. vol. 41, no.3, pp. 1109-1111, 2005.

[12] A.P.S. Baghel, S.K. Shekhawat, S.V. Kulkarni and I. Samajdar, "Modeling of dynamic hysteresis for grain-oriented laminations using a viscosity-based modified dynamic Jiles-Atherton model," Physica B : Condensed Matter, Vol. 448, pp. 349-353, 1 September 2014.

[13] D. Nitzan, "Computation of flux switching in magnetic circuits," IEEE Trans. Magn., vol. MAG- 1, no. 3, pp. 222-234, 1965.

[14] N. Sadowski, N. J. Batistela, J. P. A. Bastos, and M. Lajoie-Mazenc, "An Inverse Jiles-Atherton Model to Take Into Account Hysteresis in Time-Stepping Finite-Element Calculations," IEEE Trans. Magn. vol. 38, no.2, pp. 797-800, 2002.

[15] D. C. Jiles, "Modelling the effects of eddy currents losses on frequency dependent hysteresis in electrically conducting media," IEEE Trans. Magn., vol. 30, pp. 4326-4328, Nov. 1994. 